\title{
Commuters route choice behaviour
}

\author{
R. Selten ${ }^{\text {a }}$, T. Chmura ${ }^{\text {b,* }}$, T. Pitz ${ }^{\text {b }}$, S. Kube ${ }^{\text {c }}$, M. Schreckenberg ${ }^{d}$ \\ a Laboratory of Experimental Economics, Adenauerallee 24-42, 53113 Bonn, Germany \\ ${ }^{\mathrm{b}}$ Department of Economics, Shanghai Jiao Tong University, Shanghai 200052, PR China \\ c University of Karlsruhe, Department of Economics, Kollegium IV an Schloß, 76128 Karlsruhe, Germany \\ $\mathrm{d}$ Physics of Transport and Traffic University of Duisburg-Essen, Campus Duisburg, \\ Lotharstr. 1, 47048 Duisburg, Germany
}

Received 17 August 2004

Available online 6 May 2006

\begin{abstract}
The paper reports laboratory experiments with a two route choice scenario. In each session 18 subjects had to choose between a main road $M$ and a side road $S$. The capacity of $M$ was larger. Feedback was given in treatment I only on the subjects' own travel time and in treatment II on travel time for $M$ and $S$. The main results are as follows:

- Mean numbers on $M$ and $S$ are near to pure equilibrium.

- Fluctuations persist until the end of the sessions.

- The total number of changes is significantly greater in treatment I.

- Subjects' road changes and payoffs are negatively correlated.

- A direct response mode results in more changes for bad payoffs whereas a contrary response mode shows opposite reactions.

- Simulations of an extended payoff sum learning model fits the main results of the statistical evaluation of the data.
\end{abstract}

(C) 2006 Elsevier Inc. All rights reserved.

JEL classification: C88; C91; C92; C15; D83; R40; R41

Keywords: Travel behaviour; Information in intelligent transportation systems; Day-to-day route choice; Laboratory experiments; Payoff sum model

\footnotetext{
* Corresponding author.

E-mail address: chmura@uni-bonn.de (T. Chmura).
} 


\section{Introduction}

Understanding individual travel behaviour is essential for the design of Advanced Traveller Information Systems (ATIS), which provide real-time travel information, like link travel times. However, the response of road users to information is still an open question. It is not clear whether more information is beneficial. Drivers confronted with too much information may become oversaturated in the sense that information processing becomes too difficult and users develop simple heuristics to solve the situation. Drivers may also overreact to information and thereby cause additional fluctuations. Thus, the behaviour of the drivers has to be incorporated in traffic forecasts. ATIS can reduce fluctuations only if behavioural effects are correctly taken into account. However, are there general patterns of decision behaviour with respect to information available?

A number of experiments on route choice behaviour (e.g. Bonsal, 1992; Mahmassani and Liu, 1999) have already been reported. Here, we focus on the route choice in a generic two route scenario, which already has been investigated in literature (e.g. Iida et al., 1992). However, our aim is to present experiments with a large number of periods and with sufficiently many independent observations for meaningful applications of non-parametric significance tests.

The route choice game explored here has some similarities to experimental games in literature. The route choice game has a multitude of pure equilibria. In each of these equilibria, the payoff for each player is 10 . Therefore, one can regard the route choice game as a coordination problem in which the players have to coordinate their route choices which they distribute among the alternatives in an equilibrium fashion. Nevertheless, the situation is very different from that of the coordination games in experimental literature (Van Huyck et al., 1990). There, the players have to coordinate on an equilibrium at which everybody chooses the same pure strategy.

Market entry games (Rapoport et al., 2002; Erev and Rapoport, 1998) are another kind of games found in experimental literature. Usually in these games, the players have the choice either to enter a market or to stay out. The payoff for entering the market is a decreasing function of the number of entrants. The payoff for staying out is a constant opportunity cost. One may say that the route choice game is similar to a market entry game with two markets instead of one. However, the players do not have the choice to stay out of both markets.

Coordination games and market entry games typically have been played over a small number of periods (e.g. Van Huyck et al., 1990). It has been shown (Berninghaus and Erhart, 2001) that in coordination games extending over 100 periods, the behaviour is very different. In the minimum effort game, it converts to the best equilibrium instead of the worst. Traffic situations are best modelled as frequently repeated games. Therefore, our experiments are run over 200 periods.

If one wants to investigate results of day-to-day route choice which can be transferred to more realistic environments, it is necessary to explore individual behaviour in an interactive experimental set-up. Does behaviour converge to equilibrium? Does more feedback reduce fluctuations? What is the structure of individual responses to recent experiences? Our experimental study tries to throw light on these questions.

\section{Experimental set-up}

Subjects are told that in each of the 200 periods, they have to make a choice between a main $\operatorname{road} M$ and a side road $S$ for travelling from $A$ to $B$ (see Fig. 1).

They were told that $M$ is faster if $M$ and $S$ are chosen by the same number of people. The number of subjects in each session was 18 , mostly law and economic students from the University of Bonn. The time $t_{M}$ and $t_{S}$ depends on the numbers $n_{M}$ and $n_{S}$ of participants choosing $M$ and 


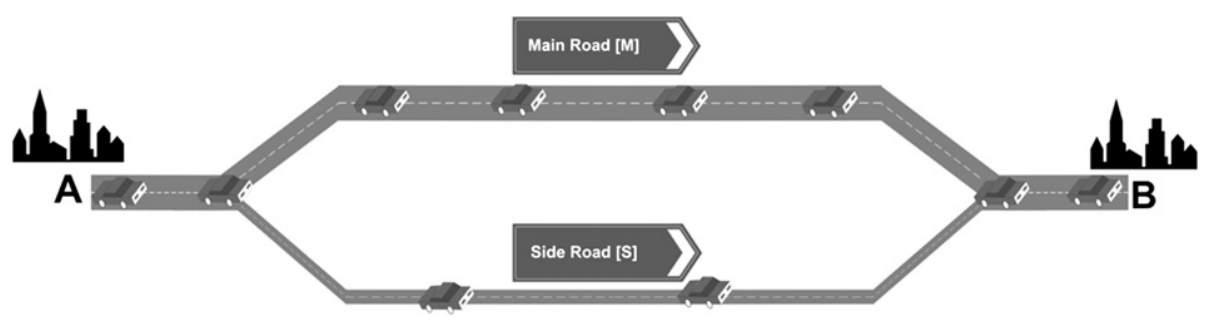

Fig. 1. Participants had to choose between a side $\operatorname{road}[S]$ and a main road $[M]$.

$S$ respectively:

$$
\begin{aligned}
& t_{M}=6+2 n_{M}, \\
& t_{S}=12+3 n_{S} .
\end{aligned}
$$

The period payoff was $40-t$ with $t=t_{M}$ if $M$ was chosen and $t=t_{S}$ if $S$ was chosen. The total payoff of a subject was the sum of all 200 period payoffs converted to money payoffs in DM with a fixed exchange rate of 0.015 DM for each experimental money unit (Taler). Additionally, every participant received a lump sum payment of 200 Taler and a show-up fee of $10 \mathrm{DM}$. One session took roughly one and a half hours.

All pure equilibria of the game are characterised by

$$
n_{M}=12 \text { and } n_{S}=6,
$$

resulting in a period payoff of 10 Taler per player. This adds up to 180 Taler per period, differing from the Pareto-optimum with 181 Taler. The Pareto-optimum can be reached by

$$
n_{M}=11 \text { and } n_{S}=7 .
$$

The game also has mixed equilibria, in particular one symmetric mixed equilibrium. In this symmetric mixed equilibrium, every player chooses the main road with probability

$$
x=\frac{58}{85}=0.682352 .
$$

This corresponds to an expected number of 12.2824 players on the main road and of 5.7176 on the side road. The computation of the symmetric mixed equilibrium can be found in Appendix C.

Two treatments have been investigated. In treatment I, the subjects received feedback at the beginning of each period after the first one about the following items:

- travel time of the last chosen route,

- last chosen route,

- payoff of the last period in Taler,

- cumulative payoff in Taler,

- the number of the current period.

In treatment II, additional feedback was provided about the travel time on the non-chosen-route in the last period. Six sessions were run with treatment I and six with treatment II. No further information was given to the subjects. 


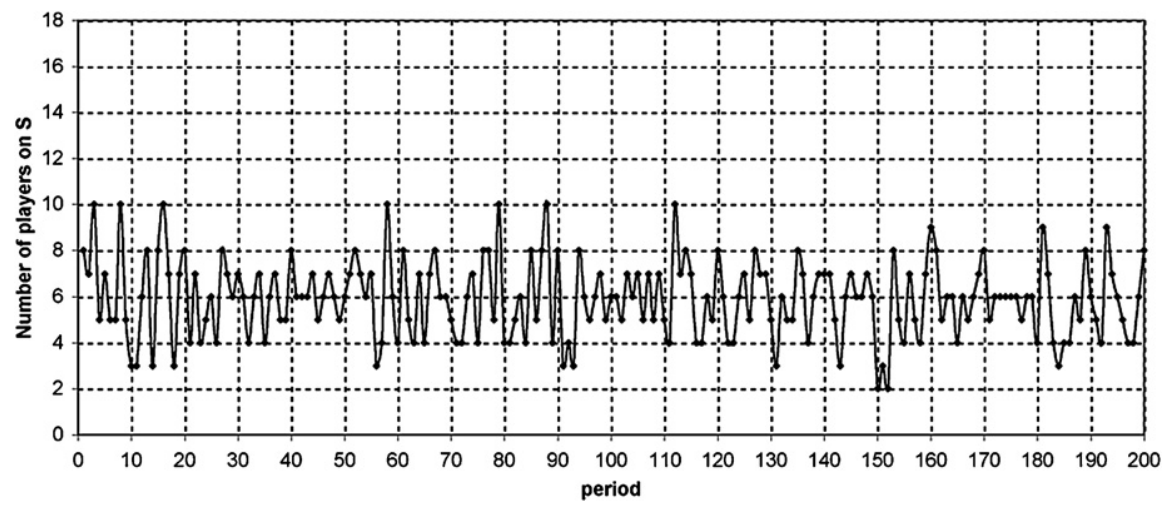

Fig. 2. Number of participants on $S$ (a typical session of treatment I).

\section{Equilibrium predictions and observed behaviour}

\subsection{Number of players on the side road $S$}

It can be seen that there is no convergence to the theoretical equilibrium. There are substantial fluctuations until the end of the session. Figure 2 shows the number of participants on $S$ in a typical session of treatment I. The same is true for all sessions of both treatments. The overall average of numbers of participants on $S$ is very near to the equilibrium prediction. In each session the median number of players on the side road $S$ is 6 . The mean number of players on the side road $S$ is 5.98 in treatment I and 6.06 in treatment II. The standard deviation of the session average from 6 is never greater than 0.17 and it is only 0.07 on average.

The fluctuations can be measured by the standard deviation of the number of participants choosing $S$ per period. This standard deviation is between 1.53 and 1.94 . With regard to these numbers, one can speak of substantial fluctuations in each of the 12 sessions.

Considering the persisting fluctuations, one might think that a mixed equilibrium is a better explanation of the data. The symmetric mixed equilibrium predicts a standard deviation of 1.98 from the mean number of participants choosing $S$ in this equilibrium. This prediction lies outside the observed range. It is shown in Appendix $\mathrm{C}$ that the symmetric mixed equilibrium grossly underestimates the mean number of players on $S$ compared to the observed number. The hypothesis that the symmetric mixed equilibrium is played can be rejected with extremely low error probability.

It is also shown in Appendix $\mathrm{C}$ that the symmetric mixed equilibrium predicts a number of road changes which is $72 \%$ higher than the actually observed number. Also on the basis of this observation, the hypothesis that the mixed equilibrium is played can be rejected with extremely low error probability. Fluctuations around the pure equilibrium seem to be a much better explanation of the data.

The fluctuations are a little larger under treatment I than under treatment II. The effect is significant. The null-hypothesis of no difference is rejected by a Wilcoxon-Mann-Whitney Test on the significance level of $5 \%$ (one-sided).

The game underlying the experiment has many pure strategy equilibrium points. In all of them, the number of participants on the side road is 6 but the set of players who choose $S$ can be any set of 6 players. The multiplicity of pure strategy equilibria poses a coordination problem which may be one of the reasons for non-convergence and the persistence of fluctuations. Feedback on 
both travel times vs. feedback on the subjects' own travel only time has the beneficial effect of reducing the fluctuations but this effect is relatively small.

\subsection{Road changes}

Figure 3 shows an example of the number of road changes as a function of time for a typical session of treatment I. Table 1 shows the mean and the standard deviation of the number of players on the side road $[S]$.

There was a negative trend in each session of treatment II. By comparison, in treatment I there were two sessions with a positive, two with a negative and two with an indifferent trend. The fluctuations are connected to the total number of road changes within one session.

The Spearman-rank-correlation between the total number of road changes and the standard deviation of the number of participants per period on $S$ is 0.795 . This is significant on the level

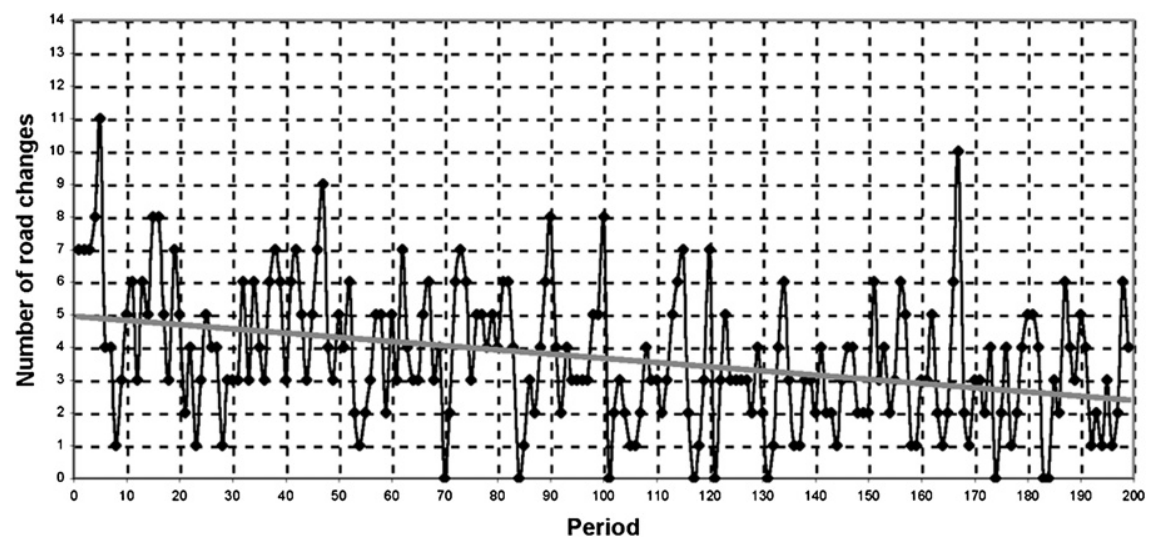

Fig. 3. Number of road changes (a typical session of treatment I).

Table 1

Mean and standard deviation of the number of players on $S$

\begin{tabular}{|c|c|c|c|}
\hline & & \multicolumn{2}{|c|}{ Number of players on $S$} \\
\hline & & $\overline{\text { mean }}$ & $\overline{\text { std. dev. }}$ \\
\hline \multirow[t]{7}{*}{ Treatment I } & session I 01 & 6.02 & 1.814 \\
\hline & session I 02 & 5.91 & 1.691 \\
\hline & session I 03 & 6.01 & 1.849 \\
\hline & session I 04 & 5.85 & 1.748 \\
\hline & session I 05 & 6.1 & 1.81 \\
\hline & session I 06 & 6.03 & 1.793 \\
\hline & treatment I & 5.98 & 1.784 \\
\hline \multirow[t]{7}{*}{ Treatment II } & session II 01 & 5.98 & 1.64 \\
\hline & session II 02 & 6.05 & 1.584 \\
\hline & session II 03 & 5.99 & 1.53 \\
\hline & session II 04 & 6.1 & 1.935 \\
\hline & session II 05 & 6.06 & 1.631 \\
\hline & session II 06 & 6.17 & 1.692 \\
\hline & treatment II & 6.06 & 1.669 \\
\hline
\end{tabular}


Table 2

Mean and standard deviation of the number of road changes

\begin{tabular}{llll}
\hline & & \multicolumn{2}{l}{ Number of road changes } \\
\cline { 3 - 4 } Treatment I & & mean & std. dev. \\
& & 5.08 & 2.298 \\
& session I 01 & 3.87 & 1.865 \\
& session I 02 & 5.16 & 1.934 \\
& session I 03 & 5.19 & 1.931 \\
& session I 04 & 5.28 & 2.391 \\
& session I 05 & 4.35 & 2.083 \\
Treatment II & session I 06 & 4.82 & 2.084 \\
& treatment I & 3.99 & 2.001 \\
& session II 01 & 3.68 & 2.039 \\
& session II 02 & 3.67 & 2.091 \\
& session II 03 & 5.19 & 2.32 \\
& session II 04 & 4.67 & 2.48 \\
& session II 05 & 4.44 & 2.044 \\
& session II 06 & 4.27 & 2.163 \\
\hline
\end{tabular}

of $1 \%$ (one sided). The median number of road changes is significantly higher in treatment I. The null-hypothesis is rejected by the Wilcoxon-Mann-Whitney Test on a level of 5\% (one-sided). The mean number of road changes under treatment $\mathrm{I}$ is also higher than under treatment II. A Wilcoxon-Mann-Whitney Test rejects the null-hypothesis only on a very weak significance level of $8.98 \%$ (one-sided).

Under treatment I, subjects who mainly choose only one of the roads feel the need to travel on the other road from time to time in order to get information on both roads. Under treatment II, there is no necessity for such information gathering. This seems to be the reason for the greater number of changes and maybe also for the stronger fluctuations under treatment I. Table 2 shows the mean and the standard deviation of the number of road changes. The mean payoffs per period in treatment I were significantly lower than in treatment II. The standard deviation of this random variable was higher in treatment I than in treatment II. In both cases a Wilcoxon-Mann-Whitney Test rejects the null-hypothesis on a significance level of 5\% (one-sided).

\subsection{Payoffs and road changes}

In all sessions, the number of road changes of a subject is negatively correlated with the subject's payoff. Figure 4 shows that the negative correlation between the payoff and the road changes is stronger in treatment II than in treatment I.

In both treatments, the Spearman rank correlations between cumulative payoffs and the number of road changes are strictly negative (see Fig. 5). The Spearman-correlation coefficients in treatment II are lower than in treatment I. A Wilcoxon-Mann-Whitney Test rejects the nullhypothesis on a significance level of 5\% (one-sided). It is observed that some players in treatment I get a payoff above average even though they have a high change rate. The reason why this effect is not so often observed in treatment II might be that the additional information about the travel time of the non chosen route was given. So it is not necessary for players to change in order to collect information. This might explain that 3 players always stayed on the main road in treatment II, nevertheless it is surprising that one player did the same in treatment I. 

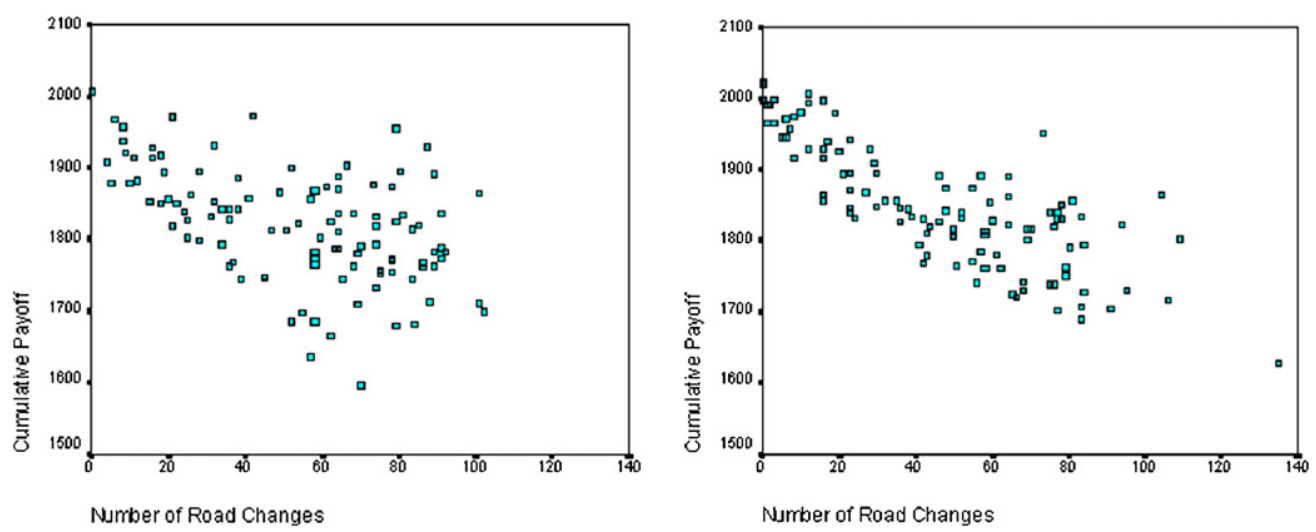

Fig. 4. Scatter diagram cumulative payoff/number of road changes for treatment I and II.

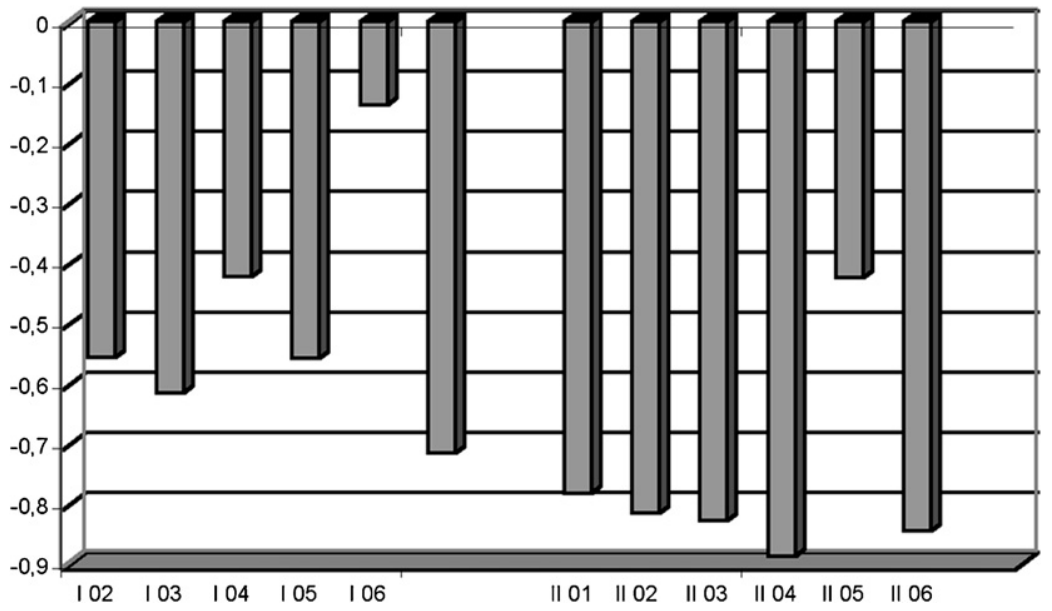

Fig. 5. Spearman-rank-correlation between cumulative payoffs and the number of road changes for each session of treatments I and II.

Even if subjects change roads in order to get higher payoffs, on average, they do not succeed in gaining this. This suggests that it is difficult to take advantage of the information provided by the feedback.

\section{Response mode}

A participant who had a bad payoff on the road chosen may change his road in order to travel where it is less crowded. We call this the direct response mode. A road change is the more probable the worse the payoff was.

The direct response mode is the prevailing one but there is also a contrary response mode. Under the contrary response mode, a road change is more likely the better the payoff was. The contrary participant expects that a high payoff will attract many others and that therefore the road chosen will be crowded in the next period. 
We do not want to assert that the response mode is a character trace. There may be people who have a general inclination towards one of the response modes but it is also possible that response modes are the results of context specific learning. The simulation model which will be described in Section 5 is based on the idea that the probabilities of using response modes are shaped by reinforcement learning.

The equilibrium payoff is 10 . Payoffs perceived as bad tend to be below 10 and payoffs perceived as good tend to be above 10. Accordingly, we classified the response of a subject as direct if the road is changed after a payoff smaller than 10 or not changed after a payoff greater than 10. An opposite response is classified as contrary. Table 3 shows the numbers of times in which a subject changes roads ( $c_{-}$for a payoff below 10 and $c_{+}$for a payoff above 10 ), or stays at the same road ( $s_{-}$for a payoff below 10 and $s_{+}$for a payoff above 10). For each subject, such a $2 \times 2$ table has been determined and a Yule coefficient $Q$ has been computed as follows.

$$
Q=\frac{c_{-} * s_{+}-c_{+} * s_{-}}{c_{-} * s_{+}+c_{+} * s_{-}}
$$

The Yule coefficient ranges from -1 to +1 . In our case, a high Yule coefficient reflects a tendency towards direct responses and a low one a tendency towards contrary responses.

In each of the four sessions, one of them in treatment I and three in treatment II, there was one player for whom no Yule coefficient could be determined since these four subjects never changed roads. These subjects are not considered in the evaluation of Yule coefficients.

The mean and the standard deviation of the Yule coefficients are shown in Table 4. Evidence for the importance of both response modes can be found in the distributions of Yule coefficients within a session. If the two response modes were not present in behaviour, one would expect

Table 3

$2 \times 2$ table for the computation of Yule coefficients

\begin{tabular}{lll}
\hline & change & stay \\
\hline payoff $<10$ & $c_{-}$ & $s_{-}$ \\
payoff $>10$ & $c_{+}$ & $s_{+}$ \\
\hline
\end{tabular}

Table 4

Mean and standard deviation of the Yule coefficients in both treatments

\begin{tabular}{llll}
\hline & & Yule coefficients Q & std. dev. \\
\cline { 3 - 4 } Treatment I & & mean & 0.654 \\
& session I 01 & 0.214 & 0.592 \\
& session I 02 & 0.373 & 0.525 \\
& session I 03 & 0.277 & 0.603 \\
& session I 04 & 0.191 & 0.584 \\
& session I 05 & 0.313 & 0.542 \\
Treatment II & session I 06 & 0.332 & 0.585 \\
& treatment I & 0.283 & 0.591 \\
& session II 01 & 0.365 & 0.536 \\
& session II 02 & 0.374 & 0.552 \\
& session II 03 & 0.308 & 0.584 \\
& session II 04 & 0.271 & 0.738 \\
& session II 05 & 0.246 & 0.557 \\
& session II 06 & 0.122 & 0.597 \\
\hline
\end{tabular}




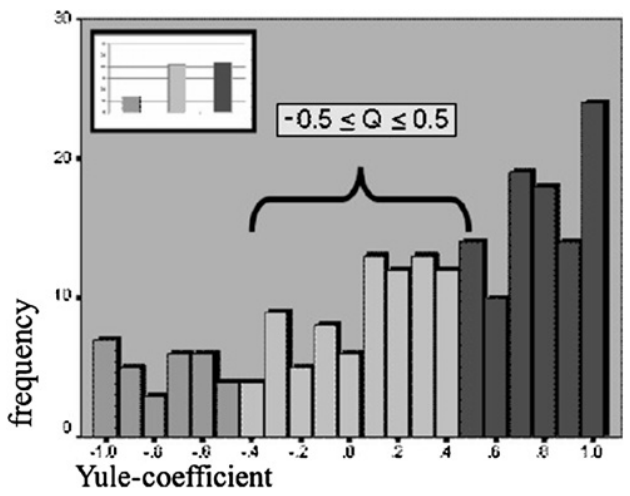

Experiments

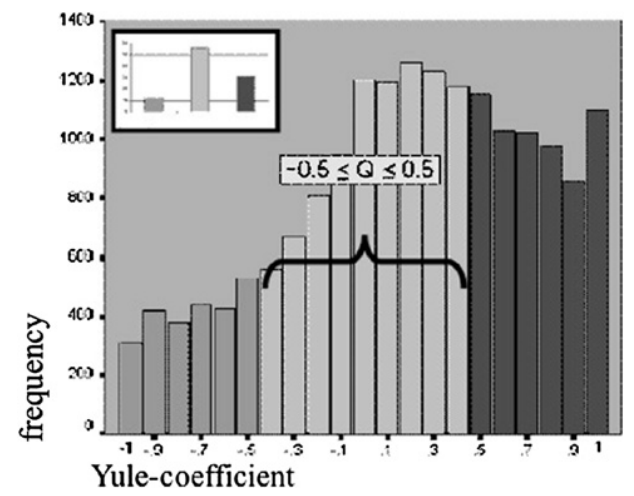

Simulations

Fig. 6. Distribution of the experimental Yule coefficients in experiments and simulations.

distributions of Yule coefficients concentrated around 0 . However, the number of subjects with extreme Yule coefficients below -0.5 or above -0.5 tends to be greater than the number of subjects with Yule coefficients in the middle range between -0.5 and +0.5 . A Wilcoxon one sample test supports this alternative hypothesis by rejecting the null-hypothesis that none of both numbers tends to be greater than the other, on the significance level of 1\% (two-sided).

If one classifies subjects with Yule coefficients above +0.5 as direct responders and subjects with Yule coefficients below -0.5 as contrary responders, then one receives $44 \%$ direct responders, $14 \%$ contrary responders and $42 \%$ unclassified subjects. The distribution of the Yule coefficients in experiments and simulations are shown in Fig. 6.

An anonymous referee suggested that the subjects in the middle range might have lower numbers of road changes than those in the extreme quartiles. However, the data show no correlation between the absolute values of the Yule coefficients and the number of road changes.

\section{Simulation of the laboratory experiments}

In order to get a deeper insight into this theoretical significance of our result, we have run simulations based on a version of a well-known reinforcement learning model, the payoff-sum model. This model already described by Harley (1981) and later by Arthur (1991) has been used extensively by Erev and Roth (1998) in the literature on experimental economics. The algorithm could be described as is shown in the enclosed box below.

We are looking at player $i$ who has to choose among $n$ strategies $1, \ldots, n$ over a number of periods $t, t=1, \ldots, T$. The probabilities with which each strategy $i$ is chosen is proportional to its "propensity" $x_{i, j}^{t}$. In period 1 , these propensities are exogenously determined parameters. Whenever the strategy $j$ is used in period $t$, the resulting payoff $a_{i}^{t}$ is added to the propensity if this payoff is positive. If all payoffs are positive, then the propensity is the sum of all previous payoffs for this strategy plus its initial propensity. Therefore, one can think of a propensity as a payoff sum.

In our experiments, negative payoffs are not impossible. This creates a difficulty for the model, since one has to exclude the case that a propensity becomes negative. If a negative payoff is obtained for the use of the strategy $x_{i}$, the absolute value of this payoff is added to all other propensities and the propensity of the strategy $i$ remains unchanged. We think that this is the simplest generalisation of the original payoff sum model which was conceived for positive payoffs only. 
Initialisation: For each player $i$ let $\left[x_{i, 1}^{1}, \ldots, x_{i, n}^{1}\right]$ be the initial propensity, where $n$ is the number of strategies, which are used in the simulations.

Period 1: Each player $i$ chooses strategy $j$ with probability $\frac{x_{i, j}^{1}}{\sum_{j} x_{i, j}^{1}}$.

Period $t+1$ : For each player $i$, let $a_{i}^{t}$ be the payoff of player $i$ in period $t$, $j$ the number of the chosen strategy in period $t$.

IF $a_{i}^{t} \geqslant 0$

$x_{i, j}^{t+1}:=x_{i, j}^{t}+a_{i}^{t}$
$x_{i, k}^{t+1}:=x_{i, k}^{t}, k \neq j$

ELSE

$x_{i, j}^{t+1}:=x_{i, j}^{t}$

$x_{i, k}^{t+1}:=x_{i, k}^{t}-a_{i}^{t}, k \neq j$

Each player $i$ chooses strategy $j$ with probability $\frac{x_{i, j}^{t+1}}{\sum_{j} x_{i, j}^{t+1}}$.

In our simulations, 18 players interact for 200 periods just like in our experiments. Each player has four strategies:

(1) Main road: This strategy simply consists in deciding to take the main road.

(2) Side road: This strategy consists in taking the side road.

(3) Direct: This strategy corresponds to the direct response mode. The payoff of a player is compared to his median payoff among his payoffs for all periods up to now. If the present payoff is lower than this median payoff, then the road is changed. If the payoff is greater than the median payoff, the player stays on the same road as before. It may also happen that the current payoff is equal to the median payoff. In this case, the road is changed if the number of previous payoffs above the median is greater than the number of previous payoffs below the median. In the opposite case, the road is not changed. In the rare cases where both numbers are equal, the road is changed with probability $1 / 2$.

(4) Contrary: A player who takes this strategy stays on the last chosen road if his current payoff is smaller than the median payoff among the payoffs for all previous periods and he changes the road in the opposite case. If the current payoff is equal to this median payoff, then he changes the road if the number of previous payoffs below the median payoff is greater than the number above the median payoff. If the numbers of previous payoffs below and above the median payoff are equal, the road is changed with probability $1 / 2$.

In the first period, only strategy one and two were available to the simulated subjects since strategy three and four cannot be applied because there is not yet a median of previous payoffs.

The strategies direct and contrary describe the response modes discussed in Section 4, but with a small difference. There, changing and staying was conditioned on how the last payoff differed from the equilibrium payoff 10 . In the experiments, the median payoff is very often at 10. However, in the simulations we did not want to build in prejudices based on theoretical values. Our simulated players base their behaviour on initial propensities and observations only. Of course, it is assumed that as in the experiments, the players get feedback about their own payoffs immediately after their choices. In the experimental treatment II, additional feedback 
Table 5

Experiments and simulations with 18 players

\begin{tabular}{lccr}
\hline & min Treatm. I \& II & Simulations & max Treatm. I \& II \\
\hline mean(\#(players[S])) & 5.85 & 5.88 & 6.17 \\
std_dev(\#(players[S])) & 1.53 & 1.65 & 1.94 \\
mean(\#(road_ch)) & 3.67 & 5.17 & 5.28 \\
mean(\#(last_road_ch)) & 154.78 & 183.73 & 190.39 \\
mean(yule) & 0.12 & 0.14 & 0.37 \\
std_dev(yule) & 0.52 & 0.60 & 0.74 \\
\hline
\end{tabular}

about the payoff on the route not chosen was given. The payoff sum model makes use of a player's own payoff only and therefore ignores the additional feedback of treatment II.

For the purposes of comparing our simulation data with the experimental data, we ignore the differences between treatments I and II which are not big anyhow.

The difficulty arises that the initial propensities must be estimated from the data. We did this by varying the initial propensities for the strategies main road and side road over all integer values from 1 to 10 and the initial propensities for the strategies direct and contrary over all integer values from 0 to 10 . We compared the simulation results with the six variables listed in Table 5. We aimed at simulation results which were between the minimum and maximum experimental results over all twelve sessions of treatments I and II. For each of the 12100 parameter combinations, we have run 1000 simulations. There was only one parameter combination which satisfied the requirement of yielding means for the six variables between the minimal and maximal experimentally observed values. This was the parameter combination $(4,3,3,2)$.

Additionally, we could show that the vector $(4,3,3,2)$ minimises the sum of normalised quadratic deviations of experimental data and simulation results of the six variables. The quadratic deviations where normalised by division by the standard deviations of the experimental results over the 12 treatments.

The numbers refer to main road, side road, direct and contrary in this order. The parameter combination is a reasonable vector of initial propensities. The players know that the capacity of the main road is greater than that of the side road and the first two parameters reflect this knowledge. It is reasonable to suppose that at least in the beginning the simple strategies main road and side road have a greater propensity sum than direct and contrary. It is surprising that a very simple reinforcement model reproduces the experimental data as well as shown by Table 5. Even the mean Yule coefficient is in the experimentally observed range, in spite of the fact that at the beginning of the simulation the behaviour of all simulated players is exactly the same. It is not assumed that there are different types of players. There are more values in the central range between -0.5 and +0.5 . However, like in Fig. 6 , many more observations are in the upper quartile than in the lowest one. This indicates that the direct response mode is much more frequent than the contrary one, in the experiments as well as in the simulations.

The distribution of the Yule coefficients suggests that during the play, the behaviour of many simulated players more and more concentrates on one strategy. This is the result of different learning histories.

It is not clear if in the actual experiments, tendencies toward direct and contrary behaviour are mere results of the learning history during the course of the game. Probably, some of the experimental subjects bring such tendencies already with them. This may be due to prior learning outside the laboratory or to inherited behavioural inclinations. One could of course try to get an 
even closer agreement with the experimental data by using a simulation model with subject heterogeneity. However, this would involve the estimation of many more parameters.

\section{Conclusion}

The study has shown that the mean numbers on both roads tend to be very near to the equilibrium. Nevertheless, fluctuations persist until the end of the sessions in both treatments. This is of particular interest in view of the fact that the experiments run over 200 periods which is unusually long and should be enough to show a tendency of convergence to equilibrium if there is one.

Feedback on both road times significantly reduces fluctuations in treatment II compared to treatment I. However, the effect is small. There is a significant rank correlation between the total number of road changes and the size of fluctuations. In treatment I, road changes may serve the purpose of information gathering. This motivation has no basis in treatment II. However, road changes may also be attempts to improve payoffs. The finding of a negative correlation between a subject's payoff and number of road changes suggests that on average such attempts are not successful.

Two response modes can be found in the data, a direct one in which road changes follow bad payoffs and a contrary one in which road changes follow good payoffs. One can understand these response modes as due to different views of the causal structure of the situation. If one expects that the road which is crowded today is likely to be crowded tomorrow, one will be in the direct response mode but if one thinks that many people will change to the other road because it was crowded today, one has reason to be in the contrary response mode. We have presented statistical evidence for the importance of the two response modes.

We have also run simulations based on a simple payoff sum reinforcement model. Simulated mean values of six variables have been compared with the experimentally observed minimum and maximum of these variables. The simulated means were always in this range. Only four parameters of the simulation model, the initial propensities, were estimated from the data. In view of the simplicity of the model, it is surprising that one obtains a quite close fit to the experimental data. The response modes direct and contrary also appear in the simulations as the result of an endogenous learning behaviour by which initially homogeneous subjects become differentiated in the course of time.

\section{Supplementary Appendixes}

Supplementary material associated with this article can be found, in the on-line version, at doi: 10.1016/j.geb.2006.03.012.

\section{References}

Arthur, W.B, 1991. Designing economic agents that act like human agents: A behavioral approach to bounded rationality. In: Papers and Proceedings of the Hundred and Third Annual Meeting of the American Economic Association. Amer. Econ. Rev. 81 (2), 353-359. May.

Berninghaus, S., Erhart, K.M., 2001. Coordination and information: Recent experimental evidence. Econ. Letters 73 , 345-351.

Bonsal, P., 1992. The influence of route guidance advice on route choice in urban networks. Transportation 19, 1-23.

Erev, I., Rapoport, A., 1998. Coordination, "magic," and reinforcement learning in a market entry game. Games Econ. Behav. 23, 146-175. 
Erev, I., Roth, A.E., 1998. Predicting how people play games: Reinforcement learning in experimental games with unique, mixed strategy equilibria. Amer. Econ. Rev. 88 (4), 848-881.

Harley, C.B., 1981. Learning in evolutionary stable strategy. J. Theoret. Biol. 89, 611-633.

Van Huyck, J.B., Battalio, R.C., Beil, R.O., 1990. Tacit coordination games, strategic uncertainty, and coordination failure. Amer. Econ. Rev. 80, 234-249.

Iida, Y., Akiyama, T., Uchida, T., 1992. Experimental analysis of dynamic route choice behaviour. Transp. Res.: Part B 26, 17-32.

Mahmassani, H.S., Liu, Y.H., 1999. Dynamics of commuting decision behaviour under advanced traveller information systems. Transp. Res.: Part C 7, 91-107.

Rapoport, A., Seale, D.A., Ordonez, L., 2002. Weighted probabilities in tacit coordination under uncertainty: Theory and evidence from market entry games. J. Risk Uncertainty 25, 21-45. 